\title{
Local approximation for contour dynamics in effectively two-dimensional ideal electron-magnetohydrodynamic flows
}

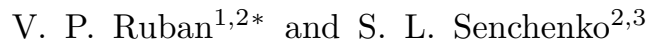 \\ ${ }^{1}$ L.D.Landau Institute for Theoretical Physics, 2 Kosygin Street, 119334 Moscow, Russia \\ ${ }^{2}$ Optics and Fluid Dynamics Department, Risø National Laboratory, DK-4000 Roskilde, Denmark and \\ ${ }^{3}$ Danish Technical University, Department of Physics, DK-2800 Lyngby, Denmark
}

(Dated: October 26, 2018)

\begin{abstract}
The evolution of piecewise constant distributions of a conserved quantity related to the frozen-in canonical vorticity in effectively two-dimensional incompressible ideal EMHD flows is analytically investigated by the Hamiltonian method. The study includes the case of axisymmetric flows with zero azimuthal velocity component and also the case of flows with the helical symmetry of vortex lines. For sufficiently large size of such a patch of the conserved quantity, a local approximation in the dynamics of the patch boundary is suggested, based on the possibility to represent the total energy as the sum of area and boundary terms. Only the boundary energy produces deformation of the shape with time. Stationary moving configurations are described.
\end{abstract}

PACS numbers: $52.30 . \mathrm{Cv}$

\section{INTRODUCTION}

In this paper we consider a special class of vortical flows in plasma that correspond to the model of ideal electron magnetohydrodynamics (EMHD) (see, e.g., [1, 2, 3, 4] and references therein about EMHD and its applications). In general, we deal with ideal EMHD-flows that are effectively two-dimensional (2D) and completely determined by a single function of two spatial coordinates and the time, namely with the usual planar flows, with the axisymmetric flows having the only azimuthal component of the magnetic field, and with the flows possessing the helical symmetry of frozen-in lines of the generalized vorticity. Our purpose here is to introduce a simplified analytical description adopted for the case when the function is piecewise constant (such distributions are usually called "patches"), with a typical spatial scale between well separated the electron inertial length and the ion inertial length. In a narrow region near sharp boundary of a "patch" the density of the electric current is high, thus the boundary can be considered as a dissipationless current sheet. From analytical viewpoint, patches are the simplest examples of EMHD-flows with current sheets, in the sense that no other degrees of freedom are present in the system, except the shape of the patch. It is a well known fact that spontaneous formation of current sheets from initially smooth magnetic field configuration is a typical phenomenon in plasma dynamics, and the role of current sheets is very important. That is why it is interesting to investigate the problem of their motion, even in such highly idealized formulation as we do in this work. As an explicit instance of fundamental physical phenomena tightly linked with our analytical study, we can indicate the fast magnetic field penetration into plasmas due to the Hall effect, since in some conditions the ideal

*Electronic address: ruban@itp.ac.ru
EMHD is applicable to describe this phenomenon [5, 6. More precisely, we focus on the effect of the electron inertia on the dynamics of a narrow moving front between magnetized and unmagnetized plasma for those special highly symmetric configurations of the generalized vorticity field. It should be noted that on the mentioned scales the magnetic field is similar to the generalized vorticity field almost everywhere except the close vicinity of the sharp patch boundary, where the magnetic field is smoothed on the electron inertial length.

As known, the EMHD model approximately describes the motion of the low-inertial electron component of plasma on sufficiently short scales (below the ion inertial length), while the much heavier ion component may be considered as motionless [1]. Conditions for electrical quasi-neutrality of the plasma are assumed, which imply that the macroscopic velocity $\mathbf{v}(\mathbf{r}, t)$ of the electron flow should be sufficiently small and also all potential waves and oscillations of the plasma density should not be excited. Only vortical degrees of freedom of the system are relevant in such circumstances. With these conditions, everywhere in the space the concentration $n(\mathbf{r}, t)$ of electrons is approximately equal to the prescribed concentration $N(\mathbf{r})$ of ions, thus the density of flow of the electrons,

$$
\mathbf{j}(\mathbf{r}, t) \equiv n(\mathbf{r}, t) \mathbf{v}(\mathbf{r}, t),
$$

is almost divergence free, $\operatorname{div} \mathbf{j} \approx 0$. The role of ions is thus reduced simply to compensation of the electric charge of electrons by providing the static neutralizing background. Such flows of the electron fluid create the divergence-free field of electric current density $-e \mathbf{j}$, where $-e$ is the electron charge. Hence, the quasi-stationary magnetic field

$$
\mathbf{B}(\mathbf{r}, t)=-\frac{4 \pi e}{c} \operatorname{curl}^{-1} \mathbf{j}(\mathbf{r}, t)
$$

should be taken into account when considering forces acting upon the electron fluid. 
Important for our consideration is that in some cases dissipative effects due to finite resistivity and/or viscosity may be neglected without dramatic loss of accuracy. So we study below the conservative EMHD model. A remarkable feature of the conservative hydrodynamic-type systems is that all of them possess an infinite number of integrals of motion related to a basic physical property of fluids, the relabeling symmetry (see, e.g., [7, 8, 9, 10] and references therein for a recent discussion). This symmetry manifests itself as the freezing-in property of a divergence-free vector field (the canonical vorticity field)

$$
\boldsymbol{\Omega}(\mathbf{r}, t)=\operatorname{curl}\left(\frac{\delta \mathcal{L}}{\delta \mathbf{j}}\right)
$$

specified by the Lagrangian functional $\mathcal{L}\{n, \mathbf{j}\}$ of a model and evolving with time accordingly to the equation

$$
\boldsymbol{\Omega}_{t}=\operatorname{curl}[\mathbf{v} \times \boldsymbol{\Omega}] .
$$

For example, in the usual Eulerian hydrodynamics $\boldsymbol{\Omega}(\mathbf{r}, t)$ is simply proportional to the velocity curl. As to the ideal EMHD, it is well known fact and we will see it below once more that frozen-in is the field

$$
\begin{aligned}
\boldsymbol{\Omega}(\mathbf{r}, t) & =\operatorname{curl} m \mathbf{v}(\mathbf{r}, t)-\frac{e}{c} \mathbf{B}(\mathbf{r}, t) \\
& =\operatorname{curl} m \mathbf{v}+\frac{4 \pi e^{2}}{c^{2}} \operatorname{curl}^{-1} \mathbf{j},
\end{aligned}
$$

where $m$ is the electron mass.

Below we deal with a spatially homogeneous ion background $N=$ const, thus the flows of the electron fluid are supposed to be incompressible, $(\nabla \cdot \mathbf{v})=0$. There exist three classes (hereafter referred as $P, A, H$ ) of incompressible ideal EMHD flows having a special symmetry which allows one to describe the vorticity distribution in terms of a single scalar function $\omega$ depending on two spatial coordinates and the time. Besides the usual planar flows with

$$
\boldsymbol{\Omega}_{P}=\mathbf{e}_{z} \omega(x, y, t)
$$

these effectively two-dimensional are the axisymmetric flows with zero azimuthal velocity component,

$$
\boldsymbol{\Omega}_{A}=\left[\mathbf{e}_{z} \times \mathbf{r}\right] \omega(z, q, t),
$$

where

$$
q=\left(x^{2}+y^{2}\right) / 2
$$

and also the flows with the helical symmetry of the frozen-in vortex lines,

$$
\begin{gathered}
\Omega_{H}^{z}=\omega(x \cos K z+y \sin K z,-x \sin K z+y \cos K z, t) \\
\Omega_{H}^{x}=-K y \Omega_{H}^{z}, \quad \Omega_{H}^{y}=K x \Omega_{H}^{z}
\end{gathered}
$$

that are space-periodic along $z$-direction with the period $L^{z}=2 \pi / K$. What is important, in each of these three cases the evolution of the corresponding $\omega(u, v, t)$ is nothing else but only the transport of its level contours by $2 \mathrm{D}$ incompressible flows. The general structure of the equations of motion for the function $\omega(u, v, t)$ is

$$
\omega_{t}+\Psi_{v} \omega_{u}-\Psi_{u} \omega_{v}=0
$$

with the stream-function $\Psi(u, v, t)$ being specified by the Hamiltonian functional $\mathcal{H}_{\sigma}\{\omega(u, v)\}$ of the model, depending also on the type of symmetry $(\sigma=P, A, H)$,

$$
\Psi=\frac{\delta \mathcal{H}_{\sigma}}{\delta \omega} .
$$

We will see later that the Hamiltonians $\mathcal{H}_{\sigma}\{\omega(u, v)\}$, corresponding to the ideal EMHD, take the quadratic form

$$
\mathcal{H}_{\sigma}\{\omega(u, v)\}=\frac{1}{2} \int \omega(u, v) \hat{G}_{\sigma} \omega(u, v) d u d v
$$

where the nonlocal linear operators $\hat{G}_{\sigma}$ possess smoothing properties like the usual two-dimensional $\Delta^{-1}$ operator. Therefore the flows with discontinuous piecewise constant distributions of the function $\omega(u, v)$ are possible. From Eqs.(9-10) it follows that the shape $\{u(\xi, t), v(\xi, t)\}$ of boundary of such a patch with $\omega=$ $\omega_{0}=$ const (here $\xi$ is an arbitrary longitudinal parameter), evolves in accordance with the equation

$$
u_{t} v_{\xi}-u_{\xi} v_{t}=\frac{1}{\omega_{0}} \frac{\partial}{\partial \xi}\left[\frac{v_{\xi}\left(\delta \mathcal{H}^{*} / \delta u\right)-u_{\xi}\left(\delta \mathcal{H}^{*} / \delta v\right)}{u_{\xi}^{2}+v_{\xi}^{2}}\right],
$$

where $\mathcal{H}^{*}\{u(\xi), v(\xi)\}$ is the energy of the patch expressed through its shape. The purpose of this work is to study the motion of the patches in $(u, v)$-plane for all three kinds of the geometric symmetry. Wee will extensively use the fact that in EMHD the kernels $G_{\sigma}\left(u_{1}, v_{1} ; u_{2}, v_{2}\right)$ of the operators are exponentially small if the distances between $\left(u_{1}, v_{1}\right)$ and $\left(u_{2}, v_{2}\right)$ are much longer than an internal length parameter of the problem, the inertial electron skin depth $d=\left(m c^{2} / 4 \pi e^{2} n\right)^{1 / 2}$. For comparatively large patches, this property makes possible to single out the local boundary term in the Hamiltonian, which is responsible for the evolution of the patch shape, while the main area term in the approximate local Hamiltonian only results in a uniform motion of the patch without changing the shape.

\section{CANONICAL FORMALISM FOR EMHD}

For convenience and self-consistency, now we reproduce briefly derivation of the EMHD equations, following the canonical formalism adopted for fluids as described in Refs. 8, 10. As the start point, let us consider the microscopic Lagrangian of a system of electrically charged point particles that can be written up to the second order on $v / c$, as it is given in the famous book by Landau and 
Lifshitz [1],

$$
\begin{aligned}
& \mathcal{L}_{\text {micro }}=\sum_{a} \frac{m_{a} \mathbf{v}_{a}^{2}}{2}-\frac{1}{2} \sum_{a \neq b} \frac{e_{a} e_{b}}{\left|\mathbf{r}_{a}-\mathbf{r}_{b}\right|}+\sum_{a} \frac{m_{a} \mathbf{v}_{a}^{4}}{8 c^{2}} \\
& +\frac{1}{4 c^{2}} \sum_{a \neq b} \frac{e_{a} e_{b}}{\left|\mathbf{r}_{a}-\mathbf{r}_{b}\right|}\left\{\mathbf{v}_{a} \cdot \mathbf{v}_{b}+\left(\mathbf{v}_{a} \cdot \mathbf{n}_{a b}\right)\left(\mathbf{v}_{b} \cdot \mathbf{n}_{a b}\right)\right\}(13)
\end{aligned}
$$

where $\mathbf{r}_{a}(t)$ are the positions of the point charges $e_{a}$, $\mathbf{v}_{a}(t) \equiv \dot{\mathbf{r}}_{a}(t)$ are their velocities, $\mathbf{n}_{a b}(t)$ are the unit vectors in the direction between $e_{a}$ and $e_{b}$. The first double sum in Eq. (13) corresponds to the electrostatic interaction, while the second double sum describes the magnetic interaction via quasi-stationary magnetic field. It is very important that for a system with macroscopically large number of particles the magnetic energy can be of the same order (or even larger) as the kinetic energy given by the first ordinary sum in Eq.(13), while the terms of the fourth order on the velocities are often negligible. Generally speaking, a large part of plasma physics is governed by this Lagrangian, at least in the cases when the velocities of particles are non-relativistic and the free electro-magnetic field is not excited significantly. Obviously, different physical problems need different procedures of macroscopic consideration of this system. The most accurate (and the most complicated) would be a kinetic description. However, for our purposes it is sufficient to apply more simple and naive procedure of the hydrodynamical averaging, that gives less accurate description of the system in terms of the concentration $n(\mathbf{r}, t)$ of electrons and the density $\mathbf{j}(\mathbf{r}, t)$ of their flow, that satisfy the continuity equation

$$
\frac{\partial n}{\partial t}+\operatorname{div} \mathbf{j}=0
$$

Neglecting all dissipative processes that take place due to collisions of the particles (though on this step we strongly reduce applicability of the following conservative EMHD model), and considering the ions as macroscopically motionless, we derive from Eq. (13) the following Lagrangian functional of the electron fluid on the given static ion background with the macroscopic concentration $N(\mathbf{r})$ :

$$
\begin{aligned}
& \mathcal{L}_{e}\{n, \mathbf{j}\}=\int d \mathbf{r}\left[m \frac{\mathbf{j}^{2}}{2 n}-\varepsilon(n)\right] \\
&-\frac{e^{2}}{2} \iint \frac{d \mathbf{r}_{1} d \mathbf{r}_{2}}{\left|\mathbf{r}_{1}-\mathbf{r}_{2}\right|}\left\{n\left(\mathbf{r}_{1}\right)-N\left(\mathbf{r}_{1}\right)\right\}\left\{\left(n\left(\mathbf{r}_{2}\right)-N\left(\mathbf{r}_{2}\right)\right\}\right. \\
&+\frac{e^{2}}{4 c^{2}} \iint \frac{d \mathbf{r}_{1} d \mathbf{r}_{2}}{\left|\mathbf{r}_{1}-\mathbf{r}_{2}\right|}\left[\mathbf{j}\left(\mathbf{r}_{1}\right) \cdot \mathbf{j}\left(\mathbf{r}_{2}\right)\right. \\
&\left.+\frac{\left(\mathbf{j}\left(\mathbf{r}_{1}\right) \cdot\left\{\mathbf{r}_{1}-\mathbf{r}_{2}\right\}\right)\left(\mathbf{j}\left(\mathbf{r}_{2}\right) \cdot\left\{\mathbf{r}_{1}-\mathbf{r}_{2}\right\}\right)}{\left|\mathbf{r}_{1}-\mathbf{r}_{2}\right|^{2}}\right],(15
\end{aligned}
$$

where the internal energy $\varepsilon(n)$ of the electron fluid takes into account energy of the thermal disordered motion of electrons and also the microscopic-scale-concentrated part of the electro-magnetic energy. It should be kept in mind that $\varepsilon(n)$ depends also on the specific entropy but we suppose the flows isentropic.
Since we are interested below in relatively slow vortical motion of the electron fluid, when all possible potential waves and oscillations are not excited significantly, so the quasi-neutrality condition $n \approx N$ is well satisfied, it is possible to neglect the second line in the expression (15), as well as variation of $\varepsilon(n)$. Thus, for the EMHD, which describes this special dynamical limit of the system (15), we have the Lagrangian

$$
\mathcal{L}_{n}\{\mathbf{j}\}=\int\left[m \frac{\mathbf{j}^{2}}{2 n}+\frac{4 \pi e^{2}}{c^{2}} \frac{\left(\operatorname{curl}^{-1} \mathbf{j}\right)^{2}}{2}\right] d \mathbf{r},
$$

where the concentration $n(\mathbf{r})$ is a prescribed function, so the density $\mathbf{j}$ of flow is divergence-free, $\operatorname{div} \mathbf{j}=0$.

In general, the equation of motion for the divergencefree flux field $\mathbf{j}$ has the form (compare with [10])

$$
\frac{\partial}{\partial t} \operatorname{curl}\left(\frac{\delta \mathcal{L}\{\mathbf{j}\}}{\delta \mathbf{j}}\right)=\operatorname{curl}\left[\frac{\mathbf{j}}{n} \times \operatorname{curl}\left(\frac{\delta \mathcal{L}\{\mathbf{j}\}}{\delta \mathbf{j}}\right)\right] .
$$

In the particular case for EMHD this yields

$$
\begin{aligned}
& \frac{\partial}{\partial t}\left(\operatorname{curl} \frac{m \mathbf{j}}{n}+\frac{4 \pi e^{2}}{c^{2}} \operatorname{curl}^{-1} \mathbf{j}\right) \\
& \quad=\operatorname{curl}\left[\frac{\mathbf{j}}{n} \times\left(\operatorname{curl} \frac{m \mathbf{j}}{n}+\frac{4 \pi e^{2}}{c^{2}} \operatorname{curl}^{-1} \mathbf{j}\right)\right]
\end{aligned}
$$

One can consider the canonical vorticity field

$$
\boldsymbol{\Omega} \equiv \operatorname{curl}\left(\frac{\delta \mathcal{L}_{n}}{\delta \mathbf{j}}\right)
$$

and the Hamiltonian functional

$$
\left.\mathcal{H}\{\boldsymbol{\Omega}\} \equiv\left\{\int\left(\frac{\delta \mathcal{L}_{n}}{\delta \mathbf{j}} \cdot \mathbf{j}\right) d \mathbf{r}-\mathcal{L}_{n}\right\}\right|_{\mathbf{j}=\mathbf{j}\{\boldsymbol{\Omega}, n\}} .
$$

Using the relation

$$
\mathbf{j}=\operatorname{curl}\left(\frac{\delta \mathcal{H}}{\delta \boldsymbol{\Omega}}\right)
$$

it is possible to rewrite the equation of motion (17) in the form

$$
\boldsymbol{\Omega}_{t}=\operatorname{curl}\left[\operatorname{curl}\left(\frac{\delta \mathcal{H}}{\delta \boldsymbol{\Omega}}\right) \times \frac{\boldsymbol{\Omega}}{n}\right]
$$

that emphasizes the freezing-in property of the canonical vorticity.

Hereafter we consider the case $n=$ const and use dimensionless variables, with all length scales normalized to the ion inertial length. The expression for the EMHD Hamiltonian can be written then in the following form

$$
\mathcal{H}\{\boldsymbol{\Omega}\}=\frac{1}{2} \iint G\left(\left|\mathbf{r}_{1}-\mathbf{r}_{2}\right|\right)\left(\boldsymbol{\Omega}\left(\mathbf{r}_{1}\right) \cdot \boldsymbol{\Omega}\left(\mathbf{r}_{2}\right)\right) d \mathbf{r}_{1} d \mathbf{r}_{2},
$$

with the Green's function

$$
G(r)=\frac{\exp (-r / \lambda)}{4 \pi \lambda^{2} r}
$$




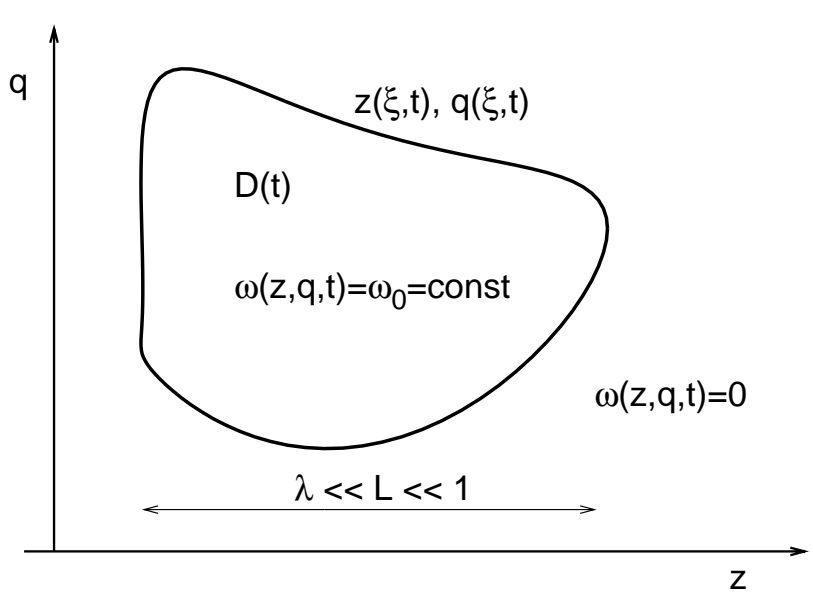

FIG. 1: Sketch of generalized vorticity patch in axisymmetric flows.

where $\lambda=\sqrt{m / M}$ is a small parameter, the ratio of the electron inertial length to the ion inertial length. It should be noted that the EMHD model in this simplest form, without taking into account the ion motion, is applicable only on scales below the ion inertial length. A more accurate model differs by another Green's function in the double integral (23): instead of the expression (24) with effectively finite radius of interaction, one has to use the modified Green's function, with infinite radius of interaction (see, e.g., [12] for more detail)

$$
\tilde{G}(r)=\frac{1}{4 \pi}\left(\frac{\exp (-r / \lambda)}{\lambda^{2} r}+\frac{1}{r}\right) .
$$

However, in this work we deal with the function (24), since our goal is to construct a local approximate model. Thus, a typical size $L$ of vortex structures must be in the limits $\lambda \ll L \ll 1$, in order the contribution to the Hamiltonian from the second term in Eq.(25) to be small in comparison with the contribution from the first one.

\section{LONG-SCALE LOCAL APPROXIMATION IN CONTOUR DYNAMICS}

\section{A. Axisymmetric flows}

Let us consider effectively 2D flows, for instance, the axisymmetric flows (6). The dynamics of the function $\omega(z, q, t)$ is determined by Eqs.(9-10), where $\mathcal{H}_{A}\{\omega\}=$ $(1 / 2 \pi) \mathcal{H}\left\{\omega\left[\mathbf{e}_{z} \times \mathbf{r}\right]\right\}$. For long-scale flows, the Green's function (24) in the double integral (23) is almost the same as the $\delta$-function. Therefore, large vortex patches like that shown in the Fig.1, with constant $\omega$ and sharp boundaries, can be approximately described by a local Hamiltonian broken onto two parts — the bulk energy

$$
\mathcal{H}_{A}^{D}=\frac{1}{2} \int_{D} \Omega^{2} r d r d z=\omega_{0}^{2} \int_{D} q d q d z,
$$

and a "surface" energy originated by the effect of nonlocality near the boundary. In leading order the boundary term can be calculated as if we have locally a $1 \mathrm{D}$ configuration with the jump $\Omega=\omega_{0} \sqrt{2 q}$ in the vorticity field. In such a $1 \mathrm{D}$ case, the additional energy per unit area of the boundary is simply

$$
-\frac{\Omega^{2}}{2 \cdot 2} \int_{0}^{\infty} e^{-\zeta / \lambda} d \zeta=-\frac{\Omega^{2} \lambda}{4}
$$

that gives the surface energy of the patch in the axisymmetric flows,

$$
\begin{aligned}
\mathcal{H}_{A}^{\partial D} & =-\frac{\lambda \omega_{0}^{2}}{4} \oint_{\partial D} r^{2} \cdot r \sqrt{(d z)^{2}+(d r)^{2}} \\
& =-\frac{\lambda \omega_{0}^{2}}{2} \oint_{\partial D} q \sqrt{2 q(d z)^{2}+(d q)^{2}} .
\end{aligned}
$$

Let us for simplicity take $\omega_{0}=1$. Using the explicit expression for the Hamiltonian functional $\mathcal{H}_{A}^{*}\{z(\xi), q(\xi)\}=$ $\mathcal{H}_{A}^{D}+\mathcal{H}_{A}^{\partial D}$

$$
\mathcal{H}_{A}^{*}\{z(\xi), q(\xi)\}=\oint q z q_{\xi} d \xi-\frac{\lambda}{2} \oint q \sqrt{2 q z_{\xi}^{2}+q_{\xi}^{2}} d \xi
$$

we obtain from Eq. (12) the following equation of motion, which does not depend on choice of the longitudinal parameter $\xi$ :

$$
z_{t} q_{\xi}-z_{\xi} q_{t}=q_{\xi}+\lambda \frac{\partial}{\partial \xi}\left[\frac{1}{q_{\xi}} \frac{\partial}{\partial \xi}\left(\frac{q^{2} z_{\xi}}{\sqrt{2 q z_{\xi}^{2}+q_{\xi}^{2}}}\right)\right] .
$$

This local nonlinear equation is one of the main results of present work. It approximates the nonlocal contour dynamics in axisymmetric EMHD-flows if a typical longitudinal scale $L$ (in $(r, z)$-plane) of the contour satisfies the condition $\lambda \ll L \ll 1$.

Now we study stationary moving (along $z$-axis) configurations. Let the shape of a patch $\omega_{0}=1$ in axisymmetric flow be given (locally) by the function $z(q, t)$ (this implies the fixed choice of the longitudinal parameter in Eq.(28), $q=\xi$ ). Then the corresponding equation of motion is

$$
z_{t}(q)=1+\lambda \frac{\partial^{2}}{\partial q^{2}}\left(\frac{q^{2} z_{q}}{\sqrt{1+2 q z_{q}^{2}}}\right)
$$

Let us consider stationary moving solutions, $z_{t}=1+$ $2 \lambda C$, where $C=$ const. The shape of the boundary in such case is determined by the ordinary differential equation

$$
\frac{\partial^{2}}{\partial q^{2}}\left(\frac{q^{2} z_{q}}{\sqrt{1+2 q z_{q}^{2}}}\right)=2 C
$$

that can be easily integrated:

$$
z=\int^{q} d q \frac{\left(C q^{2}+\tilde{a} q+\tilde{b}\right)}{\sqrt{q^{4}-2 q\left(C q^{2}+\tilde{a} q+\tilde{b}\right)^{2}}}
$$




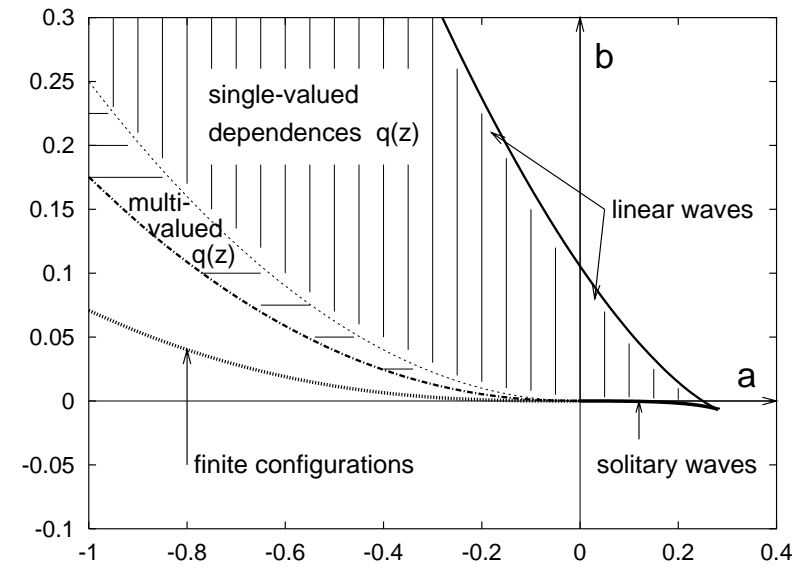

FIG. 2: The parametric plane for axisymmetric stationary solutions.

with some constants $\tilde{a}, \tilde{b}, C$. To simplify further analysis it is convenient to make the rescaling

$$
q \mapsto \alpha q, \quad z \mapsto \sqrt{\alpha} z, \quad \alpha=\frac{1}{2 C^{2}} .
$$

In these new coordinates the stationary solutions depend only on two parameters,

$$
z=\frac{1}{\sqrt{2}} \int^{q} d q \frac{q^{2}+a q+b}{\sqrt{q^{4}-q\left(q^{2}+a q+b\right)^{2}}}
$$

However, in general case the constants $a$ and $b$ may not take arbitrary values, since the expression under the square root in the denominator must be positive in some range of (positive) q. Another restriction is that selfintersections of the curves are forbidden. The parametric $(a, b)$-plane is shown in Fig.2. First, there exist $z$-periodic configurations that occupy $2 \mathrm{D}$ region in the parametric plane and correspond to axisymmetric current channels with a sharp crimped surface. On different pieces of the boundary of that region we have different types of stationary solutions. Partially the boundary is determined by the parametrically given curve (the thick solid line in Fig.2)

$$
a(\tau)=-2 \tau(\tau-3 / 4), \quad b(\tau)=\tau^{3}(\tau-1 / 2) .
$$

If the parameter $\tau$ is within the limits $3 / 8<\tau<+\infty$, then the solutions near the curve look like linear sinusoidal waves with vanishing amplitude. If $0<\tau<3 / 8$, then $z$-period of the current channels tends to infinity, and we have highly nonlinear solitary waves. For the third piece of the boundary, where self-touch of the current channels with multi-valued dependences $q(z)$ takes place, we do not have a simple analytical expression, however it can be found numerically. Second, besides the infinitely long current channels, there exist finite configurations (cross-sections of magnetic rings). For finite

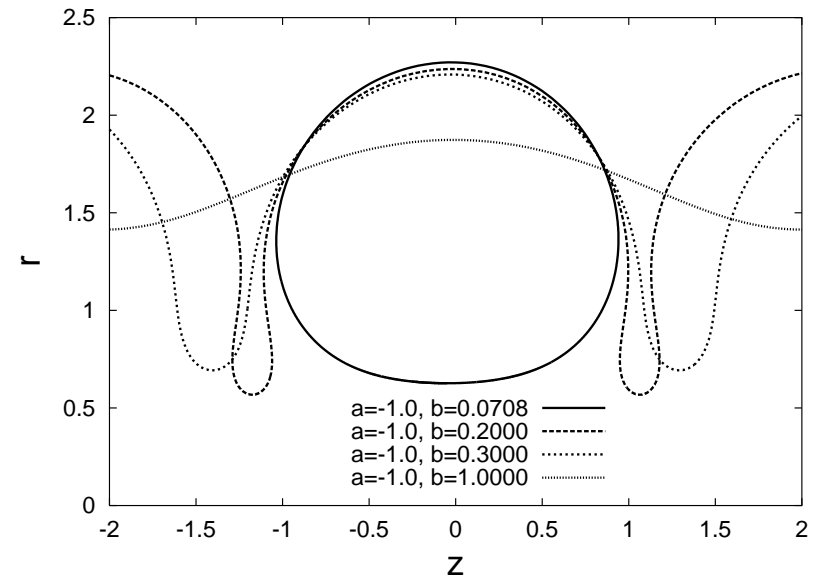

FIG. 3: Examples of axisymmetric solutions: crimped current channels and a magnetic ring.

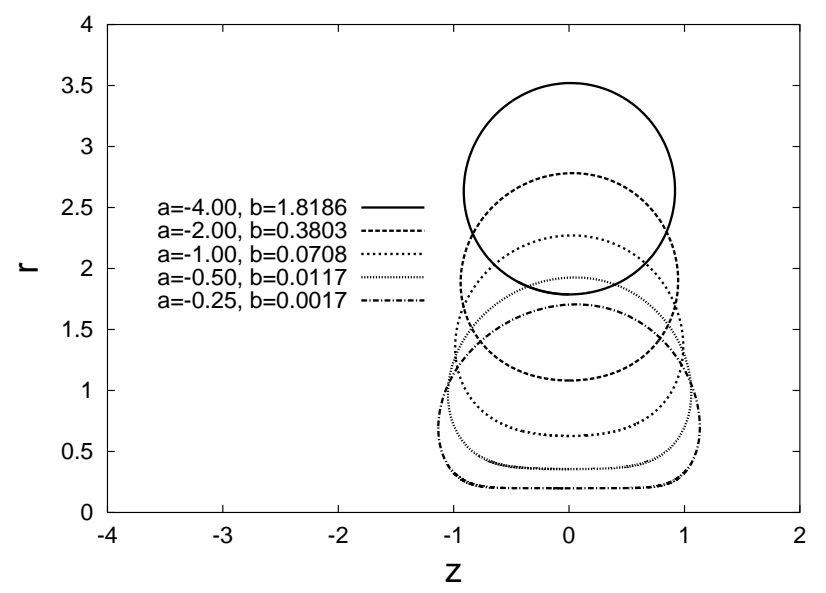

FIG. 4: Cross-sections of magnetic rings.

configurations the curves in $(z, q)$-plane must be closed, thus in this case the integral (32) between the two corresponding zeros of the denominator must be equal to zero, that gives a relation between $a$ and $b$ (the numerically calculated separate curve in Fig.21). Though the piece of the parametric curve (33) with $-\infty<\tau<0$ corresponds to nothing in the parametric plane, but the line of finite configurations approaches it from below, as $a \rightarrow 0$.

Examples of appropriate solutions are presented in Fig.3 and Fig.4. Strictly speaking, only the magnetic rings can satisfy the condition $L \ll 1$. For the infinitely long current channels, applicability of the local model is not so well justified as for the rings.

In the limit, when $a \rightarrow 0$, the "upper" part of the contour shape is the parabola when being viewed in $(q, z)$ plane (the half-circle in $(r, z)$-plane),

$$
\left(z^{2}+r^{2}\right)=2 .
$$


For the rings, the tendency towards this shape is clearly seen in the Fig, 4 , as $|a|$ decreases.

\section{B. Helical flows}

Analogously, a patch $\omega=1$ in the helical flows can be investigated. For this case the bulk energy is

$$
\begin{aligned}
\mathcal{H}_{H}^{D} & =\frac{1}{2} \int_{D}\left[1+K^{2}\left(u^{2}+v^{2}\right)\right] d u d v \\
& =\frac{1}{2} \oint_{\partial D} u d v+\frac{K^{2}}{2} \oint_{\partial D}\left(u v^{2}+u^{3} / 3\right) d v,
\end{aligned}
$$

while the boundary energy can be expressed as follows:

$$
\mathcal{H}_{H}^{\partial D}=-\frac{\lambda}{4} \oint_{\partial D}\left[1+K^{2} \mathbf{w}^{2}\right] \sqrt{(d \mathbf{w})^{2}+K^{2}(\mathbf{w} \cdot d \mathbf{w})^{2}}
$$

Here the square root is proportional to the area element of the helical tube surface.

Note that the planar flows (5) are included into this consideration, since they correspond to the case $K=0$. The boundary term in that case is simply proportional to the length of the contour that results in the integrable dynamics 13, 14 - the evolution of the boundary curvature $\kappa(l, t)$, where $l$ is the arc-length along the contour, is determined by the modified $\mathrm{KdV}$ equation,

$$
\kappa_{t}=\frac{\lambda}{4}\left(\kappa_{l l l}+\frac{3}{2} \kappa^{2} \kappa_{l}\right) .
$$

In the case $K \neq 0$, the term $\mathcal{H}_{H}^{D}$ just produces the uniform rotation of the contour with the angular velocity $\dot{\varphi}_{0}=-K^{2}$ around the origin. The shape evolution generated by $\mathcal{H}_{H}^{\partial D}$ is more convenient for analytical study in the polar coordinates (note the relation $\partial(u, v) / \partial(q, \varphi)=1)$

$$
u=\sqrt{2 q} \cos \varphi, \quad v=\sqrt{2 q} \sin \varphi,
$$

since the expression for $\mathcal{H}_{H}^{\partial D}\{\varphi(q)\}$ does not contain $\varphi(q)$ itself, but only $\partial_{q} \varphi(q)$ :

$$
\mathcal{H}_{H}^{\partial D}=-\frac{\lambda}{4} \int d q\left[1+2 K^{2} q\right] \sqrt{1 / 2 q+2 q \varphi_{q}^{2}+K^{2}}
$$

The equation of motion is

$$
\begin{aligned}
\varphi_{t} & =-\frac{\partial}{\partial q}\left(\frac{\delta\left(\mathcal{H}_{H}^{D}+\mathcal{H}_{H}^{\partial D}\right)}{\delta \varphi}\right) \\
& =-K^{2}-\frac{\lambda}{4} \frac{\partial^{2}}{\partial q^{2}}\left(\frac{2 q \varphi_{q}\left[1+2 K^{2} q\right]}{\sqrt{1 / 2 q+2 q \varphi_{q}^{2}+K^{2}}}\right) .
\end{aligned}
$$

For stationary rotation, when $\varphi_{t}=-K^{2}-C \lambda$, we have

$$
\varphi(q)=\int^{q} \frac{d q}{q} \frac{\left(C q^{2}+B q+A\right) \sqrt{1+2 K^{2} q}}{\sqrt{2 q\left(1+2 K^{2} q\right)^{2}-4\left(C q^{2}+B q+A\right)^{2}}}
$$

Here also a right choice of the constants $A, B, C$ must satisfy the condition for the curve to be closed and do not have self-intersections (see Fig.5).
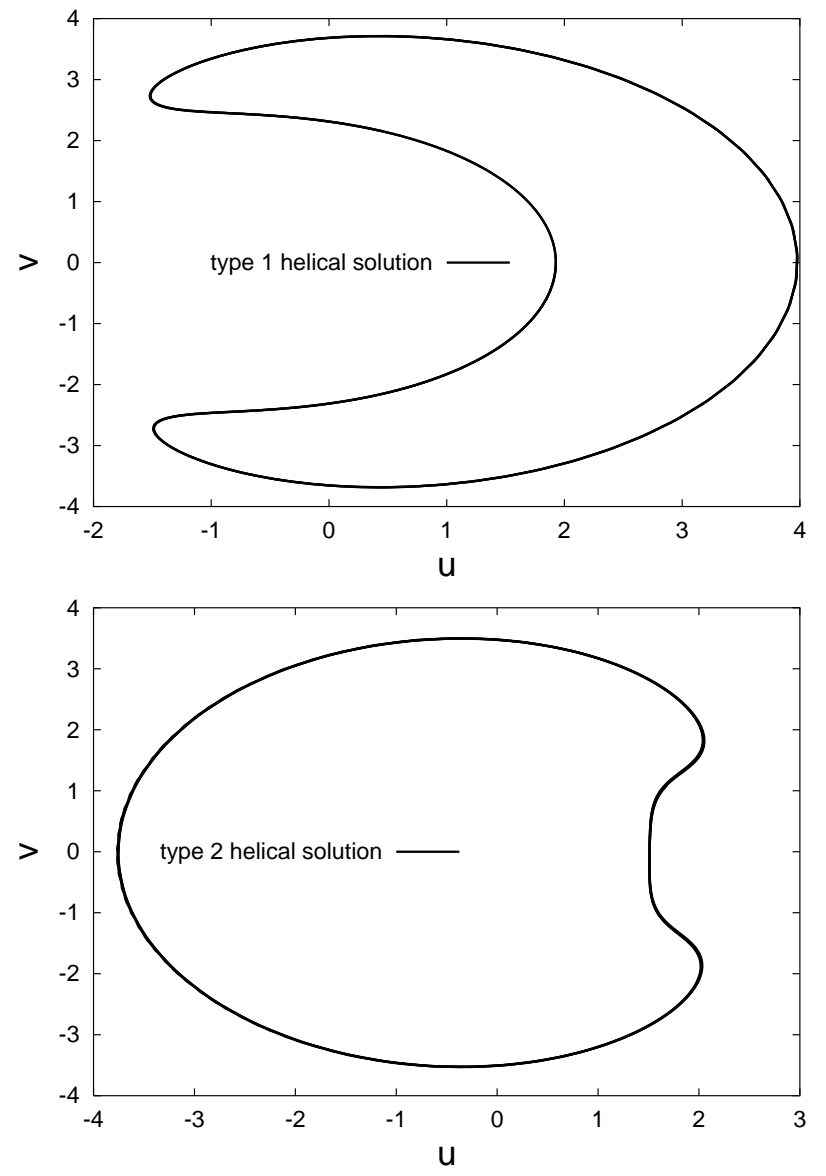

FIG. 5: Examples of stationary rotating patches in helical flows.

\section{DISCUSSION}

Thus, a local approximation for a class of effectively 2D flows in the ideal EMHD, based on the Hamiltonian formulation of the problem, has been suggested for the nonlinear contour dynamics. Stationary moving configurations with current sheets have been analytically (in integral form) found within this approximation. They describe propagation of magnetic rings, traveling waves on a crimped surface of current channels, including solitary waves, and rotating helical magnetic structures. Of course, our results are not able to explain all the important phenomena in EMHD. A payment for the relative simplicity of our local models has been the general impossibility to consider in such a manner short-scale $(\sim \lambda)$ contour perturbations, though their dynamics may have influence on the problem of stability of the stationary moving configurations. Also, the question about evolution of 3D perturbations of the magnetic rings, current channels, and/or helical structures cannot be answered in such very simplified description of the flows by moving curves in a plane. But we believe our approach useful since it develops an insight in qualitative theoretical un- 
derstanding of such complicated nonlocal theory as the EMHD. For instance, we do not see another simple and compact way how to derive equations of motion for current sheets, analogous to Eqs.(28) and (39).

\section{Acknowledgments}

These investigations were supported by the Danish Graduate School in Nonlinear Science and by the IN-
TAS (grant No. 00-00292). The work of V. R. was supported also by RFBR (grant No. 00-01-00929), by the Russian State Program of Support of the Leading Scientific Schools (grant No. 00-15-96007), and by the Science Support Foundation, Russia.
[1] A.S. Kingsep, K.V. Chukbar, and V.V. Yan'kov, in Reviews of Plasma Physics edited by B. Kadomtsev (Consultants Bureau, New York, 1990), Vol. 16, p.243.

[2] K. Avinash, S. V. Bulanov, T. Esirkepov, P. Kaw, F. Pegoraro, P. V. Sasorov, and A. Sen, Phys. Plasmas 5, 2849 (1998).

[3] D. Biskamp, E. Schwarz, A. Zeiler, A. Celani, and J. F. Drake, Phys. Plasmas 6, 751 (1999).

[4] N. Attico, F. Califano, and F. Pegoraro, Phys. Plasmas 7, 2381 (2000).

[5] A. Fruchtman, Phys. Fluids B 3, 1908 (1991).

[6] S. B. Swanekamp, J. M. Grossmann, A. Fruchtman, B. V. Oliver, and P. F. Ottinger, Phys. Plasmas 3, 3556 (1996).

[7] V.E. Zakharov and E.A. Kuznetsov, Usp. Fiz. Nauk 167, 1137 (1997) [Phys. Usp. 40, 1087 (1997)].
[8] V.P. Ruban, Zh. Eksp. Teor. Fiz. 116, 563 (1999) [JETP 89, 299 (1999)].

[9] E.A. Kuznetsov and V.P. Ruban, Phys. Rev. E 61, 831 (2000).

[10] V.P. Ruban, Phys. Rev. E 64, 036305 (2001).

[11] L.D. Landau and E.M. Lifshitz, The Classical Theory of Fields (Pergamon, Oxford, 1980) [Russian original (Nauka, Moscow, 1973)].

[12] V.P. Ruban, physics/0110023; Phys. Rev. E 65, 047401 (2002).

[13] R.E. Colgdstein and D.M. Petrich, Phys. Rev. Lett. 67, 3203 (1991).

[14] R.E. Coldstein and D.M. Petrich, Phys. Rev. Lett. 69, 555 (1992); 